ARTICLE

https://doi.org/10.1038/s41467-020-17474-0

\title{
Rhodanine-based Knoevenagel reaction and ring-opening polymerization for efficiently constructing multicyclic polymers
}

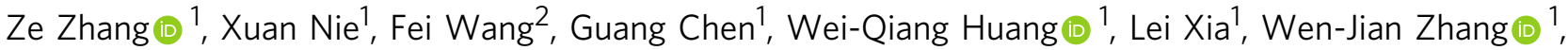

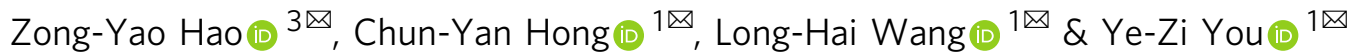

Cyclic polymers have a number of unique physical properties compared with those of their linear counterparts. However, the methods for the synthesis of cyclic polymers are very limited, and some multicyclic polymers are still not accessible now. Here, we found that the five-membered cyclic structure and electron withdrawing groups make methylene in rhodanine highly active to aldehyde via highly efficient Knoevenagel reaction. Also, rhodanine can act as an initiator for anionic ring-opening polymerization of thiirane to produce cyclic polythioethers. Therefore, rhodanine can serve as both an initiator for ring-opening polymerization and a monomer in Knoevenagel polymerization. Via rhodanine-based Knoevenagel reaction, we can easily incorporate rhodanine moieties in the backbone, side chain, branched chain, etc, and correspondingly could produce cyclic structures in the backbone, side chain, branched chain, etc, via rhodanine-based anionic ring-opening polymerization. This rhodanine chemistry would provide easy access to a wide variety of complex multicyclic polymers.

\footnotetext{
${ }^{1}$ CAS Key Laboratory of Soft Matter Chemistry, Department of Polymer Science and Engineering, University of Science and Technology of China, Hefei, Anhui 230026, People's Republic of China. ${ }^{2}$ The First Affiliated Hospital of University of Science and Technology of China, Hefei, Anhui 230001, People's Republic of China. ${ }^{3}$ The First Affiliated Hospital of Anhui Medical University and Institute of Urology, Anhui Medical University, Hefei, Anhui 230022, People's

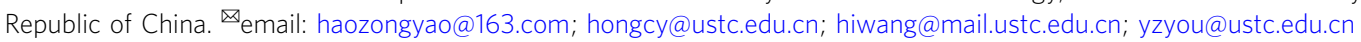


$\mathrm{n}$ the past decades, cyclic polymers have attracted a large academic attention because cyclic polymers do not contain terminal groups ${ }^{1}$, and as a result exhibit lots of unique properties including a more significant difference in the refractive index, glass-transition temperature, viscoelasticity, mechanochemistry, stability in the presence of salt and protein, and surface properties in comparison with their linear analogues ${ }^{2-8}$. Multicyclic polymers, which contain more than three cycles in one polymer molecule, have attracted considerable interest. Immunodefensins (RTD-1), oligopeptides (kalata B1), actin, and many other multicyclic biomacromolecules are widely present in the evolution of living organisms and bear extremely important life functions ${ }^{9}$. However, the synthesized polymers with complex multiple cyclic structures are still not accessible to date due to the difficulty in synthesis despite it is highly expected that such complex architectures will produce unusual or unexpected properties. Generally, the synthetic strategies towards monocyclic polymers include two categories ${ }^{10,11}$ : ring-expansion method ${ }^{12-17}$ and ring-closure method ${ }^{4,18-21}$. In ring-expansion polymerization, a cyclic initiator or nucleophile catalyst was generally used to initiate and further insert monomers into a growing (pseudo) cyclic polymer chain, and the polymerization could be performed at relatively high concentration, which makes it an appealing approach for the fast synthesis of cyclic polymers with high molecular mass in large scale. However, strict preparation conditions and minimization of side reactions are generally necessary. Another synthetic route is ring-closure technique. Although there are some needs on the concentration and time-required synthetic procedures, ring-closure method takes the advantage of conveniently utilizing the developed controlled/living polymerization strategies and efficient coupling reactions to produce cyclic polymers with designed molecular structures. Thus, the existing reports towards synthesizing complex multicyclic polymers are limited to only ring-closure strategy $22-27$ and a few cyclopolymerization strategy6,28. For example, Tezuka, etc. developed the polyaddition strategy, in which alkyne and azidecontaining cyclic precursors obtained by bimolecular ring closure using electrostatic self-assembly and covalent fixation reacted with each other to produce multicyclic polyethers ${ }^{25}$. Hong and coworkers constructed novel hyperbranched multicyclic polymers by combination of ring-closure method using ATRP and UVinduced cyclization, with self-accelerating click reaction ${ }^{22}$. Satoh, Isono and coworkers developed the ring-opening metathesis cyclopolymerizations of $\alpha, \omega$-norbornenyl end-functionalized macromonomers to prepare multicyclic polyesters and polyethers $^{28}$. Furthermore, Monteiro and Tezuka also reported the synthesis of interesting densely packed ${ }^{29}$ and knotted multicyclic polymers $^{30,31}$, respectively. However, although important progresses have been made, fast and large-scale preparation of complex multicyclic polymers via ring-expansion strategy is still unreachable.

Rhodanine is a five-membered heterocyclic compound with sulfur, nitrogen, carbonyl, and thiocarbonylthio groups in the ring (1a in Fig. 1). These heteroatoms and groups make rhodanine and its derivatives as successful scaffolds in vast fields, including dye chemistry 32,33 , the design of high-performance solar cells ${ }^{34-37}$, medicinal chemistry ${ }^{38-41}$, environmental science ${ }^{42}$. Most important, due to the five-membered cyclic structure and electron-withdrawing groups on both sides, the $-\mathrm{CH}_{2}-$ unit in the rhodanine ring is very active to aldehyde molecules via Knoevenagel reaction ${ }^{43}$ under mild conditions, which has very high efficiency and selectivity. On the other hand, Nishikubo and our groups previously have found that quaternary onium salts could catalyze the anionic ring-opening polymerization (AROP) of thiirane monomers using trithiocarbonate ${ }^{44,45}$, thioester ${ }^{46,47}$, dithiocarbonyl ${ }^{48}$, or thiourethane compounds ${ }^{49,50}$ as initiators, in which quaternary onium salt catalyzes thiirane monomer to open the ring, producing a thiolate. The thiolate further attacks the carbonyl carbon of initiator to form intermediate. After rearrangement and sequential reactions, a polythioether is obtained ${ }^{46}$. The structure and chemical properties of thiocarbonylthio groups in rhodanine are similar to trithiocarbonate, thioester, thiourethane, or dithiocarbonyl compounds. Therefore, we speculate that rhodanine could be attacked by the thiolate, resulting a cyclic polythioether by expanding the ring of rhodanine (Fig. 1).

Herein, we report rhodanine-based Knoevenagel reaction and ring-opening polymerization system, in which via rhodanine-based Knoevenagel reaction, we can easily incorporate rhodanine moieties in the backbone, side chain, hyperbranched chain, etc, and subsequently via rhodanine-based ring-opening polymerization, a
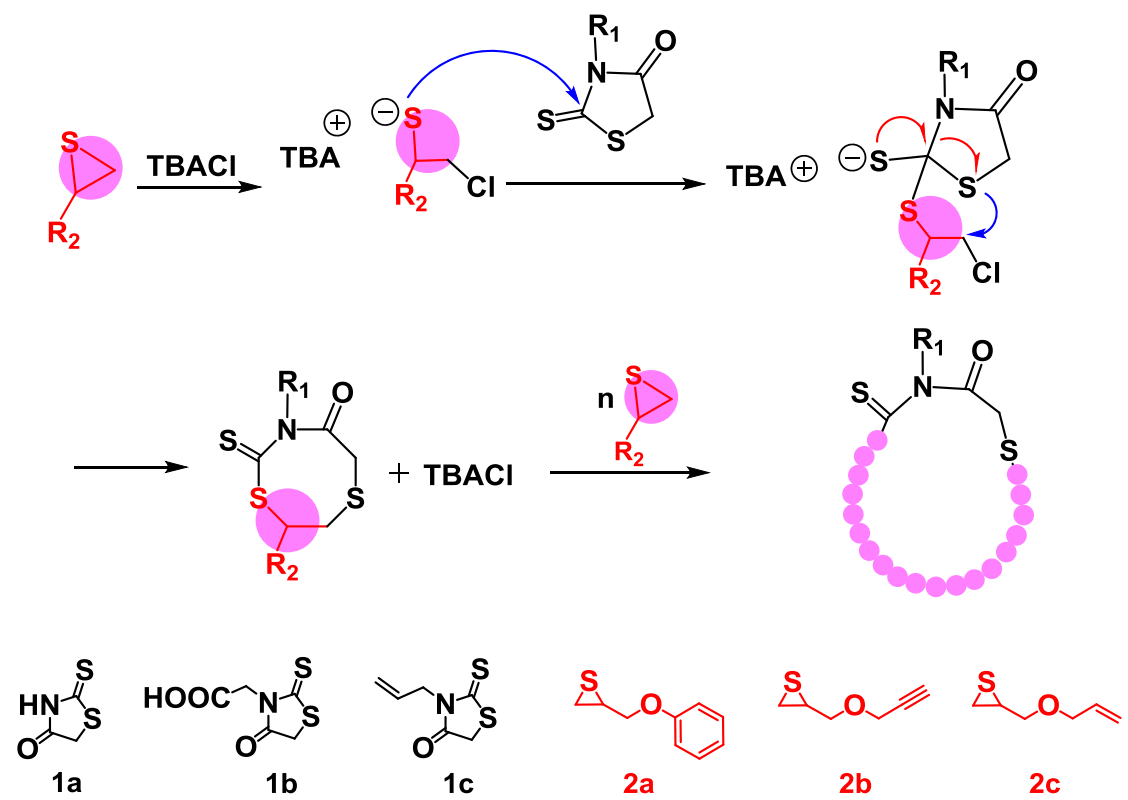

Fig. 1 Schematic illustration of the synthesis of cyclic polymer. Rhodanines initiate the anionic ring-opening polymerization (AROP) of thiiranes catalyzed by tetrabutylammonium chloride $(\mathrm{TBACl})$ at $75^{\circ} \mathrm{C}$. 
series of multicyclic polymers can be easily obtained, and the cyclic position can be easily controlled in the main chains, hyperbranched chains, and side chains. The rhodanine-based Knoevenagel reaction and ring-opening polymerization will provide a versatile scaffold towards complex multicyclic polymers.

\section{Results}

Rhodanine-based anionic ring-opening polymerization. It is reported that the ring-opening product of thiirane could easily insert into $\mathrm{C}-\mathrm{S}$ bond in $\mathrm{C}(=\mathrm{S})-\mathrm{S}$ containing molecules, such as trithiocarbonate, or dithiocarbonyl compounds $s^{44,46,48}$. Rhodanine has the highly polarized $\mathrm{C}=\mathrm{S}$ and weak $\mathrm{C}-\mathrm{S}$ in the ring, and hence, anion could attack $\mathrm{C}=\mathrm{S}$ bond easily. Therefore, rhodanine may also act as an efficient initiator for the ring-opening polymerization of thiirane. On the other hand, due to the presence of adjacent $\mathrm{C}(=\mathrm{O})-\mathrm{N}$ unit in the ring, unlike the cyclic thioester, it seems to be difficult for rhodanine to copolymerize with thiirane monomers. Based on above consideration, the ring-opening polymerization of thiirane in the presence of rhodanine was carried out, 2-(phenoxymethyl) thiirane (POMT, 2a in Fig. 1) was used as the model monomer and tetrabutylammonium chloride (TBACl) was used as the catalyst. The polymerization mixture was heated to $75^{\circ} \mathrm{C}$, and proton nuclear magnetic resonance spectroscopy $\left({ }^{1} \mathrm{H} \mathrm{NMR}\right)$ was used to trace the polymerization process. As shown in Fig. 2a and Supplementary Fig. 1, the POMT conversion increased smoothly with time, and reached $97 \%$ after $18 \mathrm{~h}$. A linear variation of $\ln \left([\mathrm{M}]_{0} /[\mathrm{M}]\right)$ with reaction time suggested the polymerization to be pseudo-first-order with respect to monomer concetration (Fig. 2a). Also, a controlled experiment was operated in the absence of rhodanine; however, there was no obvious conversion of POMT based on ${ }^{1} \mathrm{H}$ NMR trace of reaction mixture (Supplementary Fig. 2). The above experiments clearly indicate that rhodanine could initiate the ring-opening polymerization of POMT, which is similar to those ring-opening polymerizations using trithiocarbonate, or dithiocarbonyl compounds as initiator. In the ${ }^{1} \mathrm{H}$ NMR and ${ }^{13} \mathrm{C}$ NMR spectra of the resulting purified polymer (Supplementary Figs. 3, 4), all the signals corresponding to the rhodanine and POMT repeat units were clearly observed, confirming the formation of polythioether with rhodanine-derived structure in the chain. The resulting polymer was further analyzed by size exclusion chromatography (SEC) and matrix-assisted laser desorption/ionization time of flight mass spectrometry (MALDI-TOF MS). As shown in Fig. 2b, the SEC curves gradually shifted to earlier elution time with the increase of monomer conversions. After reaching $97 \%$ conversion, the polymer had dispersity $(\nexists)$ of 1.32 and number-average molecular weight $\left(M_{\mathrm{n}}\right)$ of $3100 \mathrm{~g} / \mathrm{mol}$ (black curve in Fig. 2b, entry 1 in Table 1), which was closing to the calculated value by initial ratio and conversion, illustrating that rhodanine could provide good control over the ring-opening polymerization of POMT. The cyclic structure of the obtained polymer was evidenced by MALDI-TOF MS. As shown in Fig. 2c, the signals of the polymers are separated by $166 \mathrm{Da}$, that is, the molar mass of POMT. The molecular weights obtained from individual signals agree to the molar mass sum of rhodanine, $n$ POMT, and $\mathrm{Na}^{+}$, demonstrating the successful insertion of POMT monomers into the ring of rhodanine. The above results supported our assumption, that is, rhodanine can control the ring-opening polymerization of thiirane to produce cyclic polythioether.

Based on the mechanism, a series of cyclic polythioethers have been prepared via functional rhodanines and thiirane monomers (Table 1, Supplementary Figs. 5, 6). The polymerization using $N$-substituent rhodanine derivative, rhodanine-3-acetic acid (1b in Fig. 1) as the initiator also proceeded smoothly (entry 3 in Table 1, Supplementary Fig. 7). The resulting cyclic polymer has $M_{\mathrm{n}}$ of $4600 \mathrm{~g} / \mathrm{mol}$ and $\boxplus$ of 1.29 , which was close to the calculated value by initial ratio and conversion (Supplementary Fig. 8). In the MALDI-TOF MS, the molecular weights agree to the molar mass a

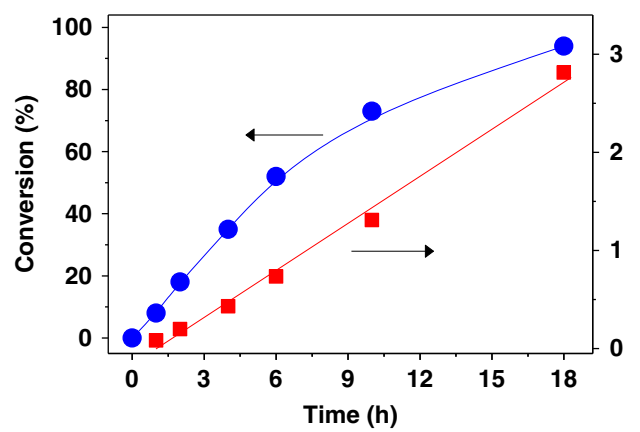

b

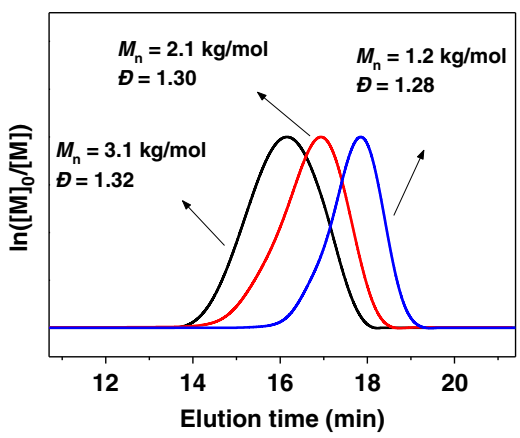

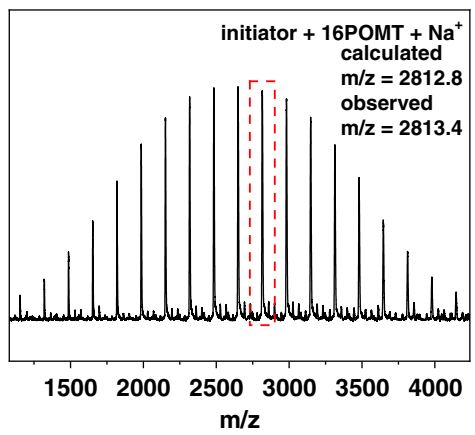

Fig. 2 Polymerization of 2-(phenoxymethyl) thiirane (POMT) using rhodanine as the initiator. a Monomer conversion and $\left.\ln ([\mathrm{M}]]_{0} /[\mathrm{M}]\right)$ versus polymerization time. $\mathbf{b}$ SEC curves for resulting cyclic polymer at different conversions. c MALDI-TOF MS spectrum of the resulting cyclic polymer.

Table 1 The results of AROP using rhodanine and its derivative as the initiators.

\begin{tabular}{|c|c|c|c|c|c|c|c|}
\hline Entry & Rhodanine $^{a}$ & Thiirane $^{b}$ & Time (h) & [Rhodanine]:[Thiirane] & Conversionc (\%) & $M_{n, S E C}{ }^{d}(g / m o l)$ & $\bigoplus^{d}$ \\
\hline 1 & $1 a$ & $2 a$ & 18 & $1: 20$ & 97 & 3100 & 1.32 \\
\hline 2 & $1 a$ & $2 b$ & 36 & $1: 40$ & 98 & 5600 & 1.23 \\
\hline 3 & $1 b$ & $2 a$ & 36 & $1: 30$ & 95 & 4600 & 1.29 \\
\hline
\end{tabular}

The polymerizations were performed at $75^{\circ} \mathrm{C}$, [thiirane $]=2.0 \mathrm{M}$.

ala and $1 \mathrm{~b}$ were shown in Fig. 1 .

${ }^{2} 2 \mathrm{a}$ and $2 \mathrm{~b}$ were shown in Fig.

CDetermined by ${ }^{1} \mathrm{H}$ NMR.

dDetermined by SEC in DMF, based on linear PSt as calibration standards. 
a
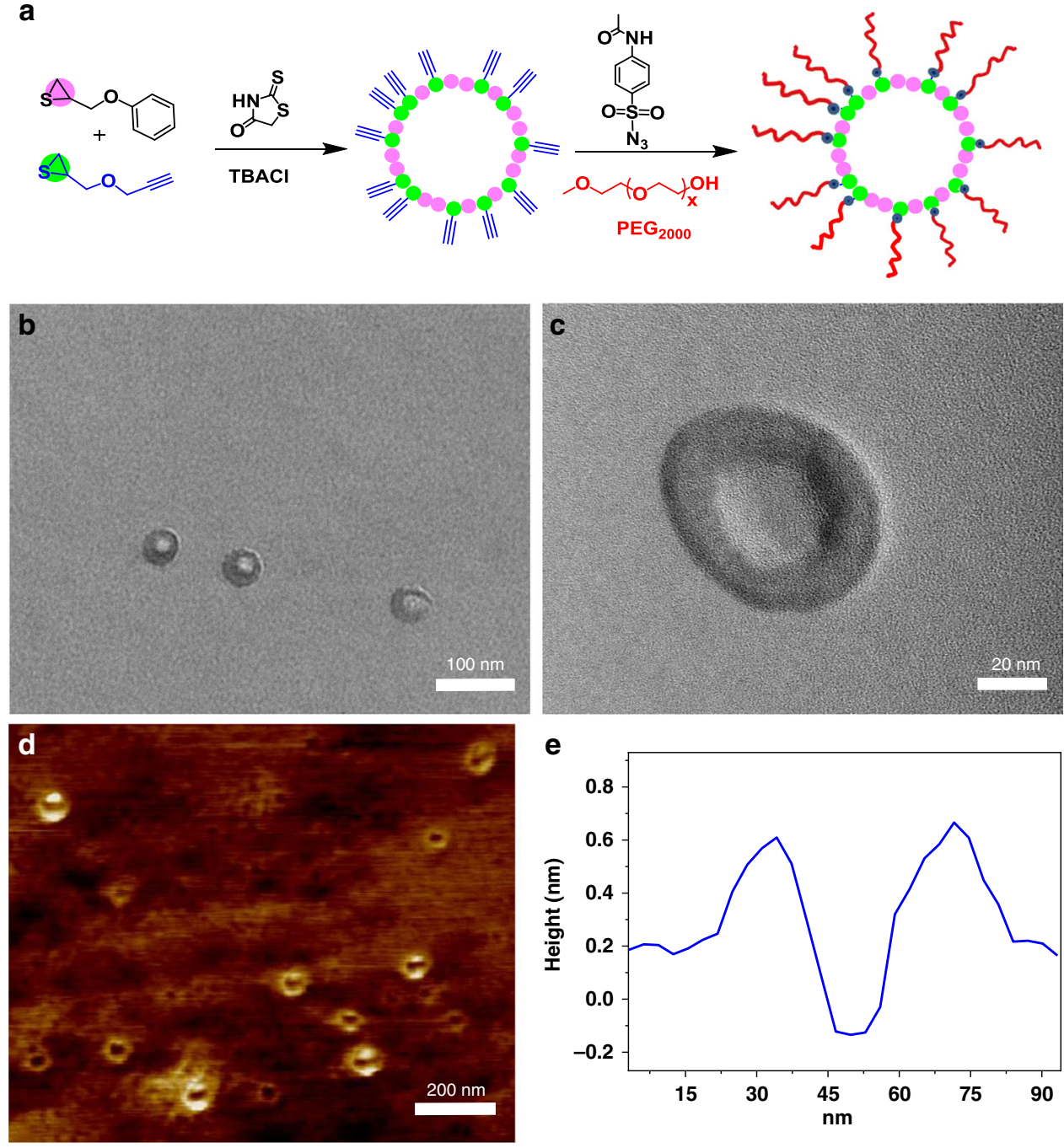

Fig. 3 Synthesis and visualization of cyclic copolymers via rhodanine-initiated AROP of thiirane monomers. a Illustration of the synthesis of cyclic graft copolymer. b TEM images of the cyclic graft copolymer. $\mathbf{c}$ HRTEM image of the cyclic graft copolymer. $\mathbf{d}$ AFM height image and e profile analysis of cyclic graft copolymer.

sum of rhodanine-3-acetic acid, $n$ POMT, and $\mathrm{Na}^{+}$, demonstrating the successful insertion of POMT monomers into the ring of rhodanine-3-acetic acid and thus the formation of cyclic polymers (Supplementary Fig. 9).

To further identify whether rhodanine can mediate the production of high-molecular-weight cyclic polymers, visualization of cyclic polymers was operated ${ }^{51}$. As shown in Fig. $3 a$, the copolymerization of POMT and alkyne-containing thiirane monomer PYMT (2b in Fig. 1) was conducted ([rhodanine]: [POMT]:[PYMT]:[TBACl] $=1 / 200 / 200 / 1)$. After $36 \mathrm{~h}$ reaction, both the conversions of POMT and PYMT reached $99 \%$, and as a result, random copolymer PPYMT- $r$-PPOMT was obtained with the $M_{\mathrm{n}}$ of $71,500 \mathrm{~g} / \mathrm{mol}$ and $Ð$ of 1.78 (entry 4 in Table 1 , Supplementary Figs. 10, 12). Then, PEG-OH $\left(M_{\mathrm{n}}=2000 \mathrm{~g} / \mathrm{mol}\right)$ were grafted on the cyclic copolymer via efficient $\mathrm{Cu}$-catalyzed azide-alkyne-hydroxyl three-component reaction (Supplementary Methods) ${ }^{52-54}$; resulting in cyclic graft polymer with the $M_{\mathrm{n}}$ of $226,500 \mathrm{~g} / \mathrm{mol}$ (Supplementary Figs. 11, 12). The visualization of the cyclic polymer molecule was carried out by transmission electron microscopy (TEM) and atomic force microscopy (AFM). According to the feed ratio and conversion, the repeat units of cyclic copolymer backbone were about 396 . Based on the bond length of each $\mathrm{C}-\mathrm{C}(0.154 \mathrm{~nm})$ and $\mathrm{C}-\mathrm{S}(0.182 \mathrm{~nm})$, the circumference and diameter of this cyclic copolymer were about 207 and $66 \mathrm{~nm}$, respectively. As shown in Fig. 3b, very obvious cyclic structures could be observed in TEM images, the outer and inner diameters of the cyclic graft polymer could be observed as $\sim 55$ and $\sim 25 \mathrm{~nm}$, respectively. High-resolution transmission electron microscopy (HRTEM) provided a clearer insight to the cyclic outline as shown in Fig. 3c. Furthermore, in AFM height image, many ring nanostructures with diameter $\sim 60 \mathrm{~nm}$ could also be observed clearly, which was another strong supporting evidence for the cyclic polymer topology (Fig. 3d, e). The above results strongly reveal that Rhodanine-AROP is a very efficient ringexpansion system for the preparation of high-yield cyclic polymers with high molecular weights via the controllable manner.

Rhodanine-based Knoevenagel reaction. Multicyclic polymers are receiving considerable interest; however, they have been largely unexplored to date because the difficulty to synthesize. Ring-expansion strategy has the advantages of rapid kinetics, high yields, and large scales. Nevertheless, most of the reported ring-expansion systems suffered the restricted initiators, uncontrollable and vulnerable polymerization process. Using ring-expansion strategy to produce various multicyclic polymers 
a

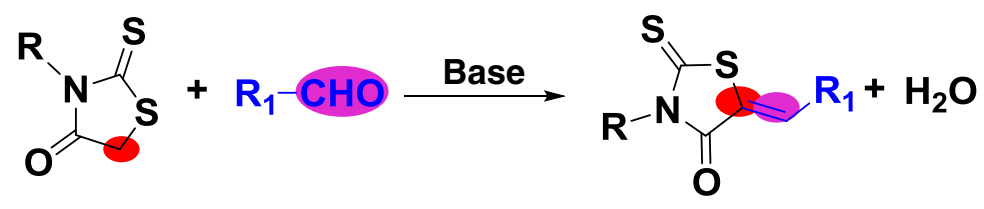<smiles>C#Cc1ccc(C=O)cc1</smiles>

b

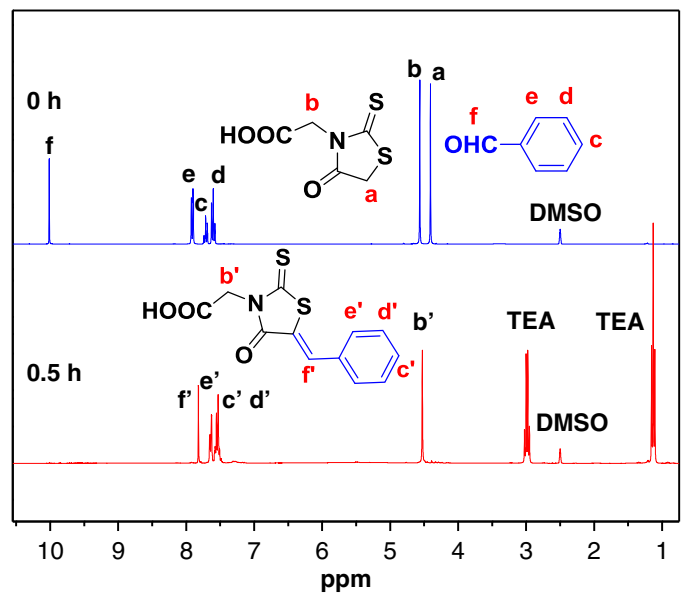

C

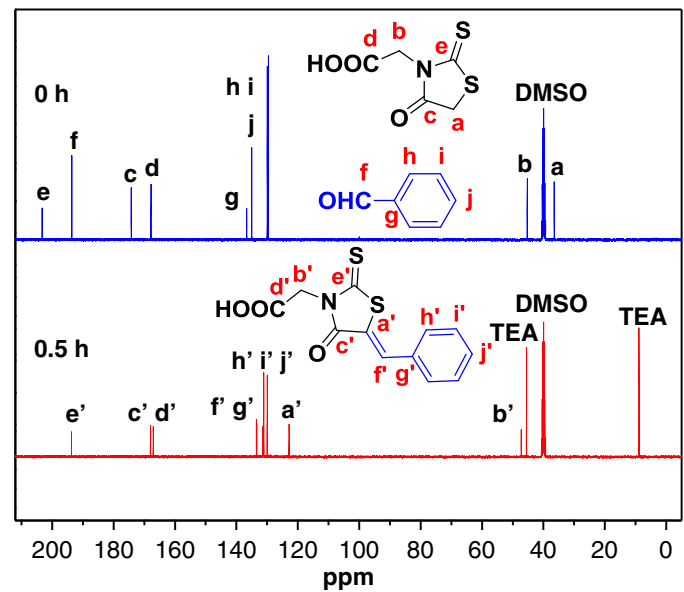

Fig. 4 Rhodanine-aldehyde (RA) Knoevenagel reaction. a RA reaction and chemicals $\mathbf{b}{ }^{1} \mathrm{H} N M R$ and $\mathbf{c}^{13} \mathrm{C}$ NMR results of RA reaction between $1 \mathrm{~b}$ and $3 a$.

is still a great challenge. If rhodanine can be polymerized, forming rhodanine-containing polymers with on-demand linear, branched or grafted structures, the formed rhodanine-containing polymers would be further used as macroinitiators for the undisturbed ring expansion, and various multicyclic topological structures would form. Therefore, the development of a rhodanine-based highly efficient reaction to produce tailorable polyrhodanines is key important for construct of various multicyclic polymers.

In 1896, German scientist, E. Knoevenagel discovered that the condensation of aldehydes or ketones with compounds containing an active methylene group in the presence of bases, resulting in the formation of ethylene derivatives ${ }^{43}$, which is widely used in the organic synthesis and in the chemical-pharmaceutical and perfume industries ${ }^{55,56}$. Generally, the Knoevenagel condensation were in moderate yields $(30-95 \%)^{57,58}$. Here, we have found that Knoevenagel reaction of rhodanine with aldehydes (RA reaction) has very high efficiency (>98\%) under mild conditions (Fig. 4a, Supplementary Fig. 13). The reaction of equimolar $\mathbf{1 b}$ and $\mathbf{3 a}$ in Fig. 4a was conducted in DMSO- $\mathrm{d}_{6}$ at $70{ }^{\circ} \mathrm{C}$ using small amount of triethylamine (TEA) as the catalyst. As shown in ${ }^{1} \mathrm{H}$ NMR spectra of reaction mixture (Fig. $4 \mathrm{~b}$ ), $1 \mathrm{~b}$ and $3 \mathrm{a}$ were mixed together without obvious reaction before TEA added. After adding TEA and heating to $70^{\circ} \mathrm{C}$, the $-\mathrm{CH}_{2}$ - protons signal of $\mathbf{1 b}$ (4.41 ppm) and the -CHO- proton signal of $\mathbf{3 a}$ (10.01 ppm) almost completely disappeared in only $0.5 \mathrm{~h}$, accompanied by the appearance of new protons for the newly produced carbon-carbon double bond (7.82 ppm). The calculated conversion was above 99\% (entry 1 in Table 2). In ${ }^{13} \mathrm{C}$ NMR (Fig. 4c),

\begin{tabular}{|c|c|c|c|c|c|}
\hline Entry & Rhodanine $^{\mathbf{a}}$ & Aldehyde ${ }^{b}$ & Solvent & Time (h) & Conversion $(\%)^{c}$ \\
\hline 1 & $1 b$ & $3 a$ & DMSO & 0.5 & 99 \\
\hline 2 & $1 b$ & $3 b$ & DMSO & 0.5 & 99 \\
\hline 3 & $1 b$ & $3 c$ & DMSO & 1.5 & 99 \\
\hline 4 & $1 b$ & $3 d$ & DMSO & 0.5 & 98 \\
\hline 5 & $1 b$ & $3 e$ & DMSO & 0.5 & 99 \\
\hline 6 & $1 c$ & $3 a$ & DMSO & 1.5 & 98 \\
\hline 7 & $1 b$ & $3 a$ & MECN & 0.75 & 98 \\
\hline 8 & $1 b$ & $3 a$ & DMF & 0.5 & 99 \\
\hline
\end{tabular}

the $-\mathrm{CH}_{2}-$ carbon signal of $\mathbf{1 b}$ was completely shifted to 122.86 ppm from $36.47 \mathrm{ppm}$ as well as the $-\mathrm{CHO}$ - carbon signal of $\mathbf{3 a}$ was also completely shifted to 133.46 ppm from $193.70 \mathrm{ppm}$, further confirming the successful formation of carbon-carbon double bond. The high-resolution mass spectrometry HR-MS analysis (Supplementary Fig. 14) also supported the exact product structure that main peak with mass value of 301.9916 corresponded to the sum of the mass of the coupling product (279.00) and the mass of $\mathrm{Na}^{+}$(22.99). Then, 1b-1c reacted with $\mathbf{3 b}-\mathbf{3 e}$ to afford a series of condensation products (Entry 2-6 in 


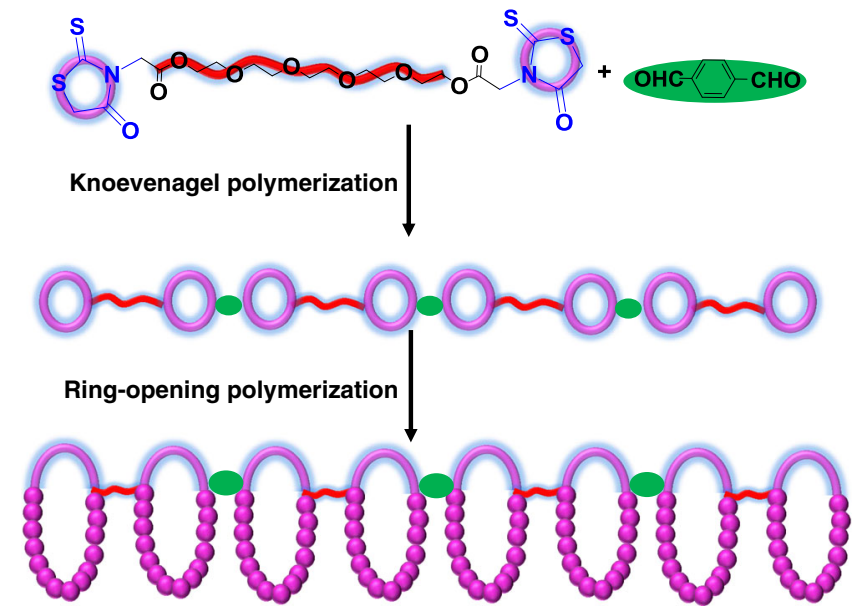

Fig. 5 Schematic illustration of the synthesis of linear multicyclic polymer. Linear polyrhodanine is produced by the Knoevenagel polymerization and acts as the efficient macroinitiator to initiate the ringopening polymerization of thiirane monomers.

Table 2, Supplementary Figs. 15-24, 29-33) with more than $98 \%$ conversions. These results reveal that the RA reaction not only has excellent orthogonality to functional groups, such as carboxyl, hydroxyl, alkynyl, alkenyl, and nitro groups, but also has very high conversions. Moreover, the RA reaction could be further conducted in other common solvents such as MeCN, DMF with nearly quantitative conversions (entry $7-8$ in Table 2, Supplementary Figs. 25-28). High efficiency, high orthogonality to various groups, high regioselectivity, easily obtained reactants, and mild condition make RA reaction a very useful tool in polymer chemistry. ${ }^{59,60}$. Additionally, as expected, the product of $\mathrm{RA}$ reaction can also be used as an initiator for ring-opening polymerization of thiiranes, resulting in cyclic polythioether with controlled molecular weight (Supplementary Figs. 34, 35).

Based on above considerations, bifunctional rhodanine compound was synthesized in one step with high yield (Supplementary Methods). Fast Knoevenagel polymerization of equimolar bifunctional rhodanine and dialdehyde (Fig. 5) was conducted at $70{ }^{\circ} \mathrm{C}$ for $3 \mathrm{~h}$, producing light-brown rhodanine-containing linear polymer with the $M_{\mathrm{n}}$ of $9100 \mathrm{~g} / \mathrm{mol}$ (Fig. $6 \mathrm{~d}$ ). The structure of this purified polymer was identified by ${ }^{1} \mathrm{H}$ NMR (Fig. 6a) and ${ }^{13} \mathrm{C}$ NMR spectra (Supplementary Fig. 36).

Multicyclic polymers with cyclic units in the backbone. Rhodanine acts as an efficient initiator for the ring-opening polymerization, forming cyclic polymers. Therefore, polyrhodanine also could act as an efficient macroinitiator for the ring-opening polymerization, forming multicyclic polymers. The linear rhodanine-containing polymer $\left(M_{\mathrm{n}}\right.$ is $\left.9100 \mathrm{~g} / \mathrm{mol}\right)$ initiated the ringopening polymerization of POMT was carried out ([rhodanine units]: $[\mathrm{POMT}]=1 / 20$ ), forming multicyclic polymers with cyclic structure in the backbone (Fig. 5). POMT conversion increased smoothly with time, and reached $83 \%$ after $16 \mathrm{~h}$ (Supplementary Fig. 37). A linear dependence of $\ln \left([\mathrm{M}]_{0} /[\mathrm{M}]\right)$ with time suggested the rate of the polymerization to be pseudo-first-order with respect to monomer concentration (Fig. 6b). The polymerization kinetics using this polyrhodanine as initiator was very similar to that using rhodanine as the initiator, suggesting the rhodanine units in the chain can still control the ring-opening polymerization of POMT. On the other hand, it was very obvious from the ${ }^{1} \mathrm{H}$ NMR spectra (Fig. 6a and Supplementary Fig. 38) of the obtained purified polymers during the polymerization process that the signals attributed to POMT could be clearly observed and gradually strengthened with the increase of polymerization time, indicating that POMT monomer has been inserted into the rhodanine units. Moreover, the carbon signal of vinyl group shifted from $124.2 \mathrm{ppm}$ to $134.1 \mathrm{ppm}$ after the ring-opening polymerization (Supplementary Fig. 39), suggesting that all the rhodanine units in the chain expanded their rings during the ring-opening polymerization. The molecule weights of the polymers were linear dependence with the monomer conversion (Fig. 6c). The produced polymers with multicyclic structure in the backbone has $M_{\mathrm{n}}$ of 23,500 and $61,800 \mathrm{~g} / \mathrm{mol}$ after 2 and $16 \mathrm{~h}$ polymerization (Fig. 6c, d), respectively, and correspondingly, each cyclic structure has $\sim 5$ and $\sim 17$ POMT units, respectively. Moreover, the size of the cyclic structure can be easily tuned via the ratio of rhodanine to POMT, and the multicyclic polymer with the $M_{\mathrm{n}}$ of $166,500 \mathrm{~g} / \mathrm{mol}$, the polymer with $\sim 43$ POMT units in each cyclic structure was prepared at the initial POMT/ rhodanine ratio of 50 (Fig. $6 \mathrm{~d}$ ).

Thermogravimetric analysis indicated that all the above polyrhodanine and multicyclic polymers showed satisfactory thermal stability with their decomposition temperature at 5 wt $\%$ weight loss ranging from 281 to $338^{\circ} \mathrm{C}$ (Supplementary Fig. 40). It is well known that glass-transition temperature $\left(T_{\mathrm{g}}\right)$ is affected by polymer architectures. Previous reports demonstrated a multicyclic polymer exhibits a higher $T_{\mathrm{g}}$ compared to its single cyclic counterpart $26,27,61$. Hence, a comparison of $T_{\mathrm{g}}$ for the obtained multicyclic PPOMT polymer with that of the corresponding single cyclic counterpart, provides extra evidence of their multicyclic architectures. A single cyclic PPOMT polymer with $\sim 100$ POMT was synthesized using the RA product (Fig. 4) as initiator. As shown in differential scanning calorimetry (DSC, Supplementary Fig. 41), $T_{\mathrm{g}}$ values of $22^{\circ} \mathrm{C}$ and $21^{\circ} \mathrm{C}$ were observed for the multicyclic polymers with average 17 and 43 POMT in each cyclic structure, respectively, which are $\sim 10^{\circ} \mathrm{C}$ higher than that of the single cyclic PPOMT polymer $\left(11^{\circ} \mathrm{C}\right)$. The higher $T_{\mathrm{g}} \mathrm{s}$ of the multicyclic polymers are attributable to the reduced free volume and conformational freedom of multicyclic architecture than single cycle 26,61 . We further tried to use TEM characterize the molecular morphology of multicyclic copolymer. Similar to Fig. 3, but with above polyrhodanine as the initiator, copolymerization of POMT and PYMT produced alkyne-containing multicyclic copolymer. Then, PEGs $\left(M_{\mathrm{n}}=1000 \mathrm{~g} / \mathrm{mol}\right)$ were grafted onto this multicyclic copolymer to ultimately obtain multicyclic molecular brush with the $M_{\mathrm{n}}$ of $978,000 \mathrm{~g} / \mathrm{mol}$. The unusual compact prayer beads-like nanostructure can be observed in TEM (Supplementary Fig. 42), suggesting the formation of interesting multicyclic structures.

Synthesis of branched multicyclic polymers. Compared with previously reported multicyclic polymer synthesis methods, this ring-expansion strategy using rhodanine-based Knoevenagel reaction and ring-opening polymerization has the obvious advantages of high reaction concentration, high yields, large of cycle numbers and relative short synthesis time. Excitingly, besides preparing linear multicyclic polymers, the rhodanine-based Knoevenagel reaction and ring-opening polymerization also enable the production of branched multicyclic polymer. As shown in Fig. 7, benzene-1,3,5-tricarbaldehyde was a trifunctional aldehyde monomer and the same difunctional monomer in Fig. 5 was used as the rhodanine monomer. To minimize gelation, dialdehyde was added as the comonomer. The polymerization was conducted with feed ratio of $[1 \mathrm{~d}]:[3 \mathrm{~g}]:[3 \mathrm{f}]=1 / 0.3 / 0.6$ to obtain rhodanine-containing branched polymer with the $M_{\mathrm{n}}$ of $7200 \mathrm{~g} / \mathrm{mol}$ and $Ð$ of 1.91 (Supplementary Fig. 43). Subsequently, this branched rhodaninecontaining polymer acted as macroinitiator for the ring-opening 


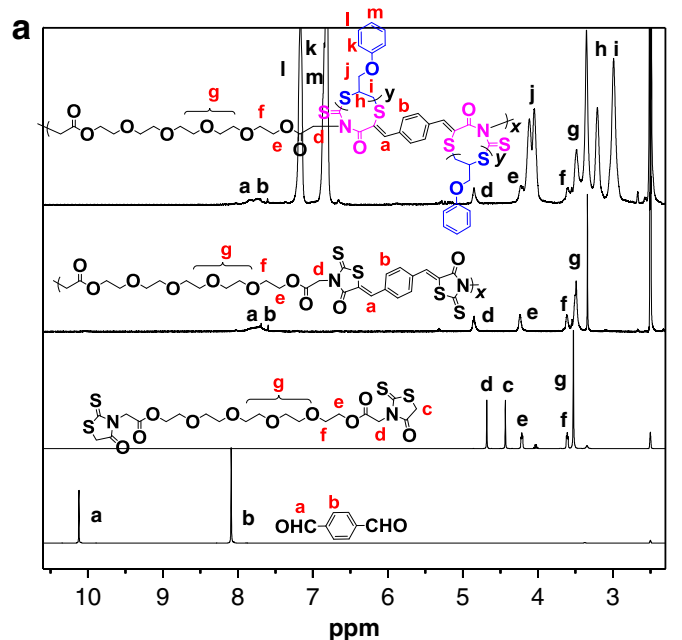

C

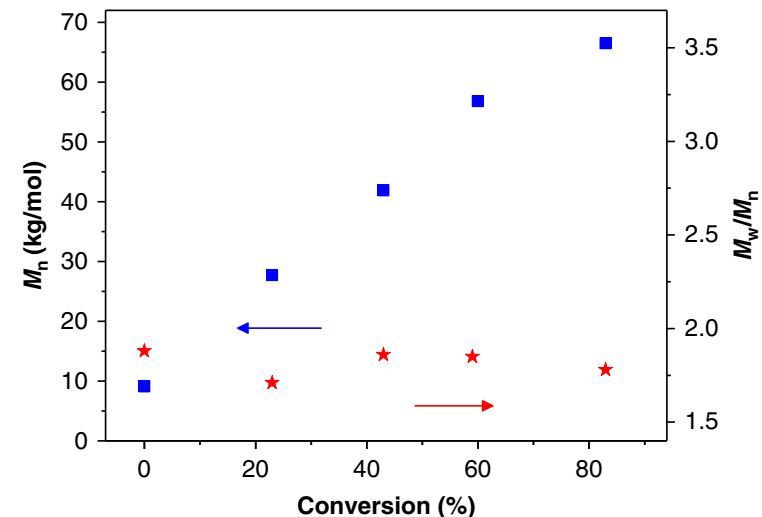

b
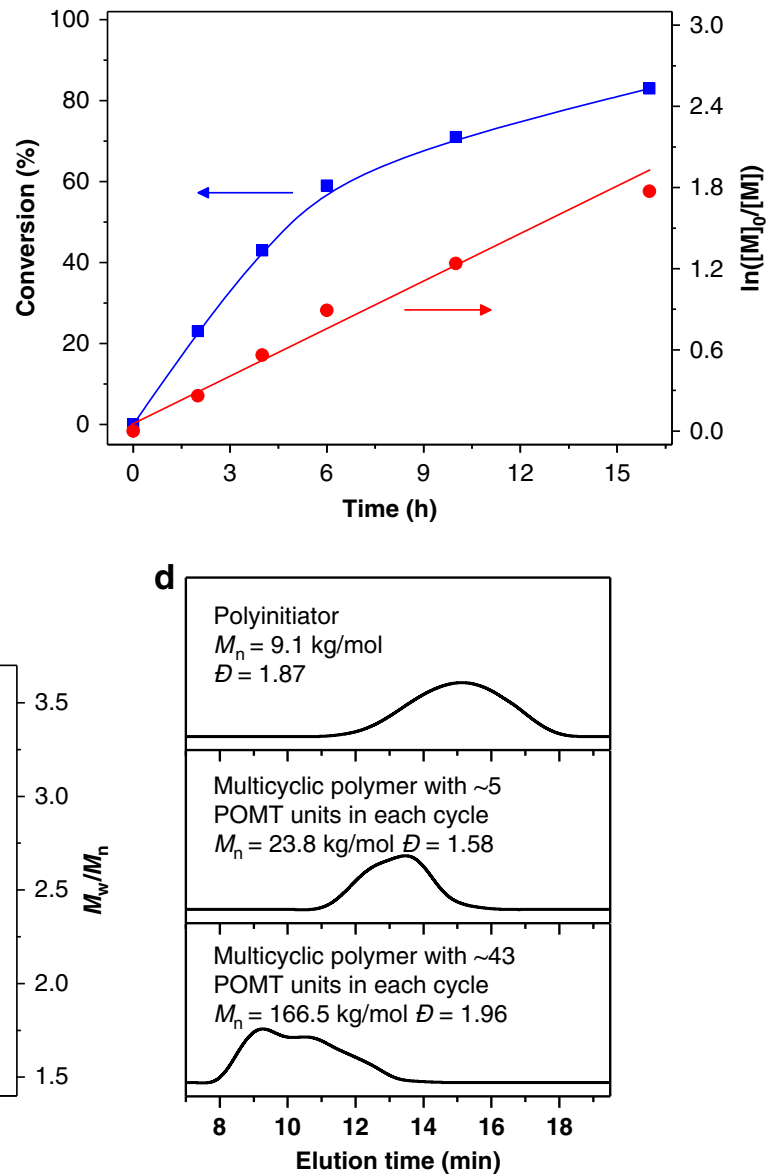

Fig. 6 Synthesis of linear multicyclic polymer with cyclic structures in the backbone. a ${ }^{1} \mathrm{H}$ NMR spectra of resulting polyrhodanine initiator and multicyclic polymers. b Monomer conversion and $\ln \left([\mathrm{M}]_{0} /[\mathrm{M}]\right)$ versus polymerization time during the synthesis of multicyclic polymer. $\mathbf{c} M_{\mathrm{n}}$ and $\bigoplus$ of multicyclic polymers at different time. d SEC curves of resulting polyrhodanine initiator and multicyclic polymers.
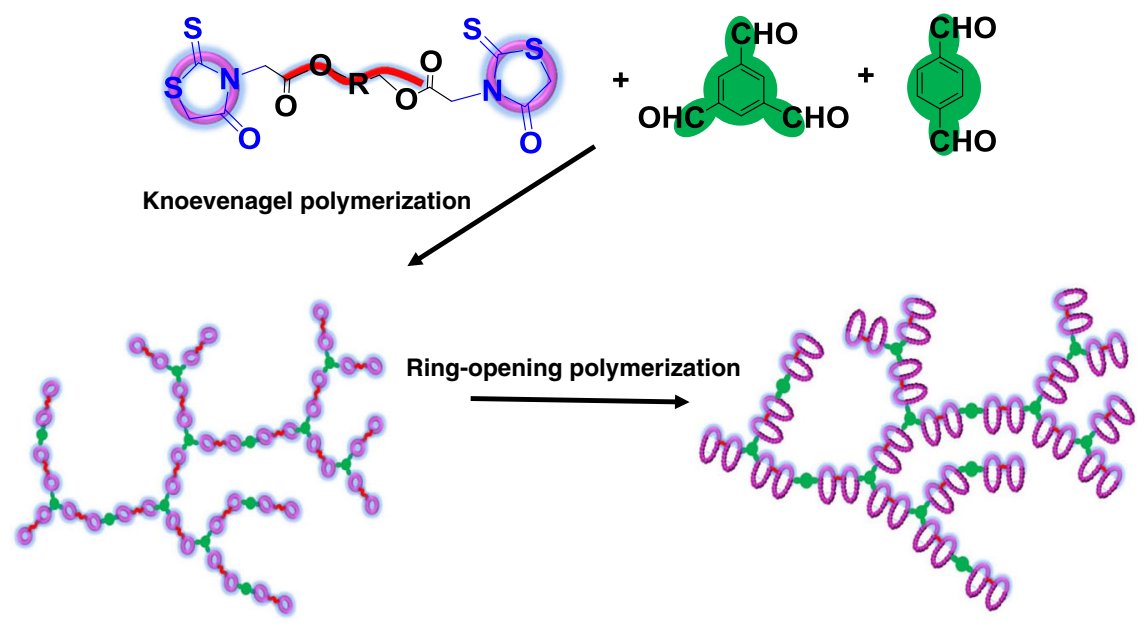

Fig. 7 Schematic illustration of the synthesis of hyperbranched multicyclic polymer. Hyperbranched polyrhodanine is produced by the Knoevenagel polymerization and acts as the efficient macroinitiator to initiate the ring-opening polymerization of thiirane monomers.

polymerization of thiirane. Alkene-containing PEMT (2c in Fig. 1) was used as a thiirane comonomer in the ring-opening polymerization. The polymerization was conducted with feed ratio of [rhodanine]:[POMT]:[PEMT] =1/15/10), resulting in alkenehanging branched multicyclic copolymer with the $M_{\mathrm{n}}$ of 27200 , $54200 \mathrm{~g} / \mathrm{mol}$ after 3 and $18 \mathrm{~h}$, respectively (Supplementary Fig. 43).
The structure was also identified by ${ }^{1} \mathrm{H}$ NMR (Supplementary Fig. 44).

Multicyclic polymers with pendant cyclic structures. The highly efficient rhodanine-based Knoevenagel reaction and ring-opening 


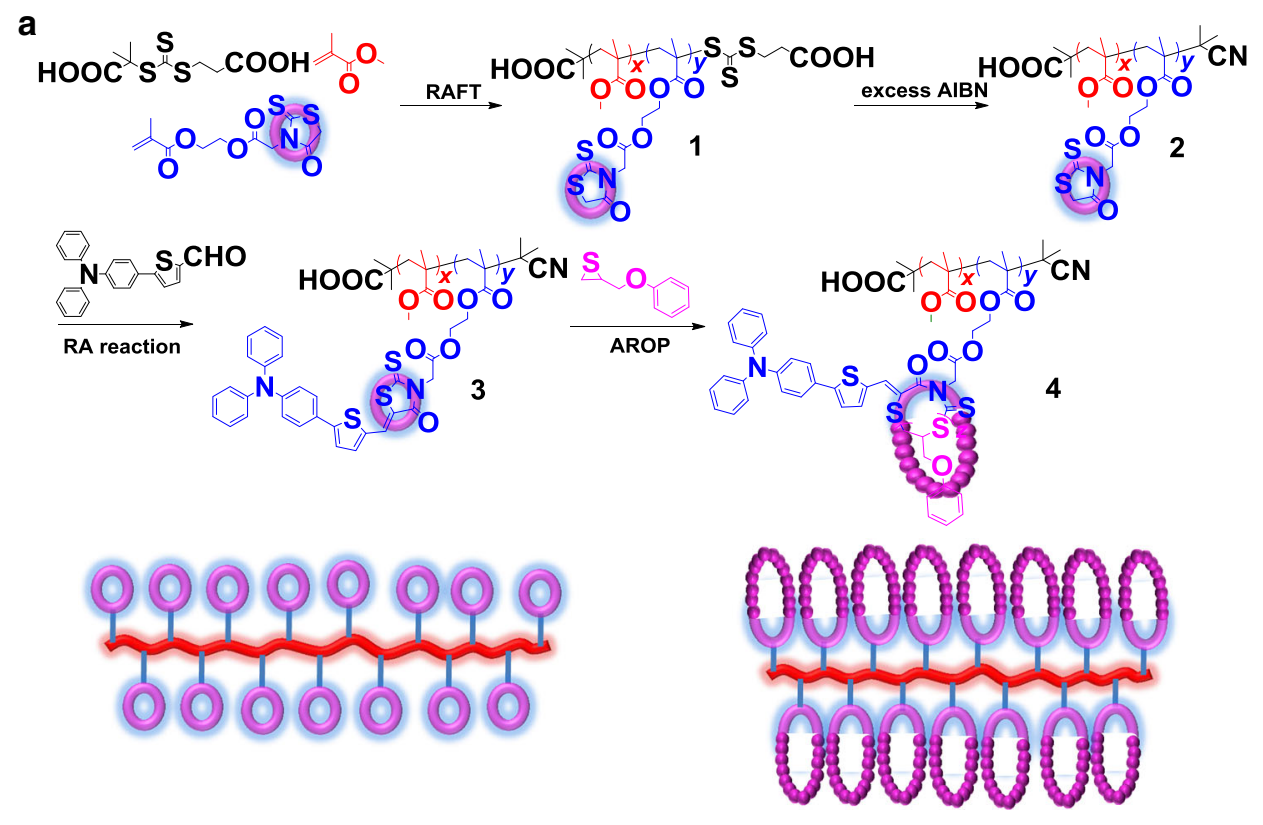

b
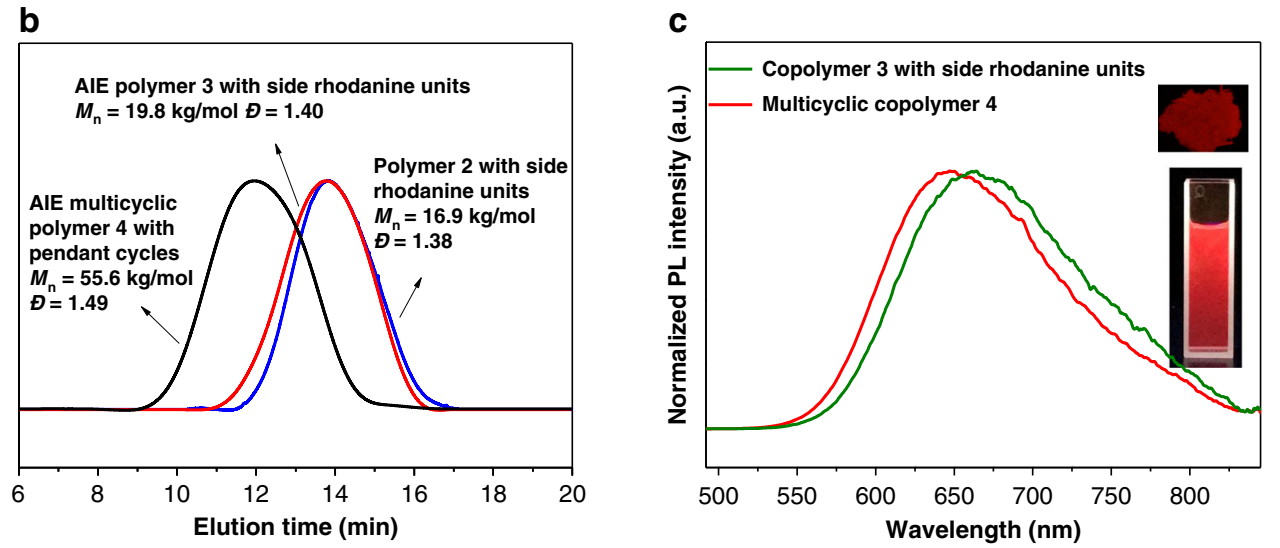

Fig. 8 Synthesizing AIE multicyclic copolymer with pendant cyclic structures. a Illustration of the synthetic route. b SEC curves for resulting polymers. c The normalized PL intensity of resulting red/near-infrared AIE copolymer 3 and multicyclic copolymer 4. Inset: fluorescent photographs of multicyclic copolymer 4 in solid state and in solution $\left(\mathrm{H}_{2} \mathrm{O}: \mathrm{THF}=95: 5\right)$.

polymerization encouraged us to further explore the synthesis of complex as well as functional multicyclic polymers. The attempt of combination of rhodanine-based ring-opening polymerization with RAFT polymerization for constructing functional multicyclic polymer with pendant cyclic structures (Fig. 8a and Supplementary Methods) was carried out. The polymerization of methyl methacrylate (MMA) and rhodanine-containing methacrylate (MRDA, Supplementary Fig. 45) in the presence of trithiocarbonate and AIBN was performed. The conversions of MMA and MRDA both reached $\sim 99 \%$ to produce copolymer 1 with $\sim 10$ rhodanine rings per chain. Then excess AIBN was added to remove the trithiocarbonate end groups, preventing it from interfering in following ring-opening polymerization ${ }^{44}$ (Supplementary Fig. 46). As shown in the ${ }^{1} \mathrm{H}$ NMR spectrum of the obtained purified copolymer 2 (Supplementary Fig. 48), all the MRDA proton signals can be clearly observed; and the integral of MMA with MRDA was very close to the initial feed ratio. Copolymer 2 had $M_{\mathrm{n}}$ of $16,900 \mathrm{~g} / \mathrm{mol}$, which was close to theoretical value as well (Fig. 8b, Supplementary Fig. 48). Above results demonstrates that rhodanine ring shows the good orthogonality to radical process, which strongly suggests rhodanine chemistry has the potential to participate in various radical polymerization methods. Then, we chose 5-(4-(diphenylamino) phenyl)thiophene-2-carbaldehyde (TTPA, Fig. 8a) to conjugate onto copolymer 2 by the efficient RA reaction to obtain copolymer 3. TTPA is a nonfluorescent molecule but usually used to construct red/near-infrared aggregation-induced emission AIEgens via electron donor-acceptor interactions ${ }^{62,63}$. In RA reaction product, the produced carbon-carbon double bond associates rhodanine ring with aromatic unit of aldehyde compound via conjugated interaction. Thus, the RA reaction product has typical donor-acceptor structure (Supplementary Fig. 47), in which rhodanine ring is acceptor unit, carbon-carbon double bond is $\pi$-bridge, and aldehyde compound is donor unit ${ }^{34,35}$. Since the RA products have donor-acceptor structures, we supposed that copolymer 3 would receive AIE property after TTPA conjugated onto rhodanine ring (Supplementary Fig. 47). As shown in Supplementary Fig. 48, the complete disappearance of rhodanine $-\mathrm{CH}_{2}$ - proton signal $(4.46 \mathrm{ppm})$ and appearance of TTPA proton signals (6.81-8.24 ppm) in the ${ }^{1} \mathrm{H}$ NMR spectrum of obtained purified copolymer 3 as well as a slight increase of $M_{\mathrm{n}}$ to $19,800 \mathrm{~g} / \mathrm{mol}$ reveal complete conjugation between rhodanine ring with TTPA (Fig. 8b). As expected, copolymer 3 interestingly emitted red/near-infrared fluorescent at the aggregated state with $\lambda_{\text {em }}$ at $660 \mathrm{~nm}$ (Fig. 8c). Then, this red/near-infrared AIE copolymer 3 further served as the macroinitiator to perform the AROP 
of POMT ([MRDA]:[POMT] $=1 / 30$ ); as the result, multicyclic copolymer 4 (Fig. 8a) was finally produced with $\sim 100 \%$ POMT conversion (Supplementary Fig. 49). The $M_{\mathrm{n}}$ of the produced copolymer $(55,600 \mathrm{~g} / \mathrm{mol}$, Fig. $8 \mathrm{~b})$ was some less than the theoretical value $(69,000 \mathrm{~g} / \mathrm{mol})$ calculated by POMT feed ratio with the average numbers of rhodanine ring in copolymer chain, which may result from smaller hydrodynamic volume of multicyclic architecture than linear analogue. Very interestingly, the multicyclic copolymer 4 still hold the AIE property and emitted red/near-infrared light at the aggregated state with $\lambda_{\mathrm{em}}$ at $645 \mathrm{~nm}$ (Fig. 8c). The absolute fluorescence quantum yield of multicyclic copolymer 4 solid was $12.08 \%$, which was comparable to existed AIEgens ${ }^{62-64}$, indicating this multicyclic polymer would be a very promising red/near-infrared luminescent material ${ }^{65}$.

\section{Discussion}

We have developed a rhodanine-based Knoevenagel reaction, in which rhodanine can react with various aldehyde reactants under moderate conditions with nearly quantitative conversions and high regioselectivity. The unique thioester-containing five-membered structure makes rhodanine, its $N$-substituent and $C$-substituent derivatives, act as initiators to control the ringopening polymerization of thiirane monomers, forming a library of cyclic polythioethers. Importantly, multicyclic polymers with cyclic structures in the backbone, branched chain, and multicyclic polymers with pendant cyclic structures were easily produced with high yields via rhodanine-based Knoevenagel reaction and ring-opening polymerization. The developed rhodanine-based chemistry opens an avenue in the design and application of functional click-like reactions as well as provides deep insight into complex multicyclic polymers for further study of structure-property relationship.

\section{Methods}

Model reaction of rhodanine-aldehyde condensation. A typical procedure is as follow: rhodanine compound $(0.2 \mathrm{mmol})$, aldehyde compound $(0.2 \mathrm{mmol})$, and TEA $(0.1 \mathrm{mmol})$ were dissolved in $0.5 \mathrm{~mL}$ DMSO-d6 and transferred into a NMR tube. Then the tube was sealed and immersed in an oil bath at $70^{\circ} \mathrm{C}$. After the certain reaction time, the conversions were analyzed by NMR measurement.

Rhodanine-initiated polymerization of thiiranes. A typical procedure is as follow: rhodanine ( $33 \mathrm{mg}, 0.25 \mathrm{mmol})$, POMT $(830 \mathrm{mg}, 5 \mathrm{mmol})$, and tetrabutylammonium chloride ( $41 \mathrm{mg}, 0.15 \mathrm{mmol}$ ) were dissolved in NMP to obtain $2.5 \mathrm{~mL}$ solution and transferred into a transparent glass tube. After two freezepump-thaw cycles, the tube was sealed and immersed in an oil bath at $75^{\circ} \mathrm{C}$. After $24 \mathrm{~h}$ reaction, the solution was precipitated into methanol several times and the product as yellow viscous solid was obtained after dried in vacuum.

Synthesis of multicyclic polymers. Synthesis of the precursor polymer: difunctional rhodanine monomer $(292 \mathrm{mg}, 0.5 \mathrm{mmol})$, terephthalaldehyde $(67 \mathrm{mg}$, $0.5 \mathrm{mmol}$ ), and TEA ( $25 \mathrm{mg}, 0.25 \mathrm{mmol}$ ) were dissolved in DMF to obtain $2.5 \mathrm{~mL}$ solution and immersed in an oil bath at $70^{\circ} \mathrm{C}$. After $3 \mathrm{~h}$ reaction, the solution precipitated into methanol several times and the product precursor polymer as light-brown solid was obtained after dried in vacuum. The precursor polymer $(72.2 \mathrm{mg}, 0.2 \mathrm{mmol}$ heterocycle), POMT (664 mg, $4 \mathrm{mmol})$, and TBACl $(56 \mathrm{mg}$, $0.2 \mathrm{mmol}$ ) were dissolved in NMP to obtain $2 \mathrm{~mL}$ solution and transferred into a transparent glass tube. After two freeze-pump-thaw cycles, the tube was sealed and immersed in an oil bath at $75^{\circ} \mathrm{C}$. After $18 \mathrm{~h}$ reaction, the solution was precipitated into methanol several times and the product as light-brown solid was obtained after dried in vacuum.

\section{Data availability}

The data that support the findings of this study are available within the article and its Supplementary Information File or from the corresponding author upon reasonable request.

Received: 17 December 2019; Accepted: 30 June 2020;

Published online: 21 July 2020

\section{References}

1. Bielawski, C. W., Benitez, D. \& Grubbs, R. H. An “endless" route to cyclic polymers. Science 297, 2041-2044 (2002).

2. Nasongkla, N. et al. Dependence of pharmacokinetics and biodistribution on polymer architecture: effect of cyclic versus linear polymers. J. Am. Chem. Soc. 131, 3842-3843 (2009).

3. Verbraeken, B. \& Hoogenboom, R. Cyclic polymers: from scientific curiosity to advanced materials for gene delivery and surface modification. Angew. Chem. Int. Ed. 56, 7034-7036 (2017).

4. Schappacher, M. \& Deffieux, A. Synthesis of macrocyclic copolymer brushes and their self-assembly into supramolecular tubes. Science 319, 1512-1515 (2008).

5. Zhang, K., Lackey, M. A., Cui, J. \& Tew, G. N. Gels based on cyclic polymers. J Am. Chem. Soc. 133, 4140-4148 (2011).

6. Chen, J. et al. Blocking-cyclization technique for precise synthesis of cyclic polymers with regulated topology. Nat. Commun. 9, 5310 (2018).

7. Cortez, M. A. et al. The synthesis of cyclic poly(ethylene imine) and exact linear analogues: an evaluation of gene delivery comparing polymer architectures. J. Am. Chem. Soc. 137, 6541-6549 (2015)

8. Morgese, G., Cavalli, E., Rosenboom, J. G., Zenobi-Wong, M. \& Benetti, E. M Cyclic polymer grafts that lubricate and protect damaged cartilage. Angew. Chem. Int. Ed. 57, 1621-1626 (2018).

9. Craik, D. J. Chemistry-seamless proteins tie up their loose ends. Science 311, 1563-1564 (2006)

10. Laurent, B. A. \& Grayson, S. M. Synthetic approaches for the preparation of cyclic polymers. Chem. Soc. Rev. 38, 2202-2213 (2009).

11. Haque, F. M. \& Grayson, S. M. The synthesis, properties and potential applications of cyclic polymers. Nat. Chem. 12, 433-444 (2020)

12. He, T., Zheng, G. H. \& Pan, C. Y. Synthesis of cyclic polymers and block copolymers by monomer insertion into cyclic initiator by a radical mechanism. Macromolecules 36, 5960-5966 (2003).

13. Kammiyada, H., Ouchi, M. \& Sawamoto, M. A study on physical properties of cyclic poly(vinyl ether)s synthesized via ring-expansion cationic polymerization. Macromolecules 50, 841-848 (2017).

14. Boydston, A. J., Holcombe, T. W., Unruh, D. A., Frechet, J. M. J. \& Grubbs, R. $\mathrm{H}$. A direct route to cyclic organic nanostructures via ring-expansion metathesis polymerization of a dendronized macromonomer. J. Am. Chem. Soc. 131, 5388-5389 (2009).

15. Shin, E. J. et al. Zwitterionic copolymerization: synthesis of cyclic gradient copolymers. Angew. Chem. Int. Ed. 50, 6388-6391 (2011).

16. Guo, L., Lahasky, S. H., Ghale, K. \& Zhang, D. H. N-heterocyclic carbenemediated zwitterionic polymerization of $\mathrm{N}$-substituted $\mathrm{N}$-carboxyanhydrides toward poly(alpha-peptoid)s: kinetic, mechanism, and architectural control. $J$. Am. Chem. Soc. 134, 9163-9171 (2012).

17. Li, H. Y., Debuigne, A., Jerome, R. \& Lecomte, P. Synthesis of macrocyclic poly(epsilon-caprolactone) by intramolecular cross-linking of unsaturated end groups of chains precyclic by the initiation. Angew. Chem. Int. Ed. 45, 2264-2267 (2006)

18. Sun, P., Chen, J. Q., Liu, J. A. \& Zhang, K. Self-accelerating click reaction for cyclic polymer. Macromolecules 50, 1463-1472 (2017).

19. Ge, Z. S. et al. High-efficiency preparation of macrocyclic diblock copolymers via selective click reaction in micellar media. J. Am. Chem. Soc. 131, 1628-1629 (2009)

20. Oike, H. et al. Designing unusual polymer topologies by electrostatic selfassembly and covalent fixation. J. Am. Chem. Soc. 122, $9592-9599$ (2000).

21. Laurent, B. A. \& Grayson, S. M. An efficient route to well-defined macrocyclic polymers via "Click" cyclization. J. Am. Chem. Soc. 128, 4238-4239 (2006).

22. Liu, C., Fei, Y. Y., Zhang, H. L., Pan, C. Y. \& Hong, C. Y. Effective construction of hyperbranched multicyclic polymer by combination of ATRP, UV-induced cyclization, and self-accelerating click reaction. Macromolecules 52, 176-184 (2019).

23. Zhao, Z. Q. et al. A dicyclic scaffold for programmed monocyclic and polycyclic polymer architectures. Macromolecules 50, 8907-8915 (2017).

24. Hossain, M. D., Jia, Z. F. \& Monteiro, M. J. Complex polymer topologies built from tailored multifunctional cyclic polymers. Macromolecules 47, 4955-4970 (2014).

25. Sugai, N. et al. Effective click construction of bridged- and spiro-multicyclic polymer topologies with tailored cyclic prepolymers (kyklo-Telechelics). J. Am. Chem. Soc. 132, 14790-14802 (2010).

26. Pipertzis, A., Hossain, M. D., Monteiro, M. J. \& Floudas, G. Segmental dynamics in multicyclic polystyrenes. Macromolecules 51, 1488-1497 (2018).

27. Hossain, M. D., Lu, D. R., Jia, Z. F. \& Monteiro, M. J. Glass transition temperature of cyclic stars. ACS Macro Lett. 3, 1254-1257 (2014).

28. Isono, T. et al. Multicyclic polymer synthesis through controlled/living cyclopolymerization of alpha,omega-dinorbornenyl-functionalized macromonomers. Macromolecules 51, 3855-3864 (2018).

29. Gavrilov, M. et al. Densely packed multicyclic polymers. ACS Macro Lett. 6 1036-1041 (2017). 
30. Kyoda, K., Yamamoto, T. \& Tezuka, Y. Programmed polymer folding with periodically positioned tetrafunctional telechelic precursors by cyclic ammonium salt units as nodal points. J. Am. Chem. Soc. 141, 7526-7536 (2019).

31. Tezuka, Y. Topological polymer chemistry designing complex macromolecular graph constructions. Acc. Chem. Res. 50, 2661-2672 (2017).

32. Marinado, T. et al. Rhodanine dyes for dye-sensitized solar cells: spectroscopy, energy levels and photovoltaic performance. Phys. Chem. Chem. Phys. 11, 133-141 (2009).

33. Insuasty, A. et al. 2-(1,1-dicyanomethylene)rhodanine A novel, efficient electron acceptor. Dyes Pigments 88, 385-390 (2011).

34. Holliday, S. et al. A rhodanine flanked nonfullerene acceptor for solutionprocessed organic photovoltaics. J. Am. Chem. Soc. 137, 898-904 (2015).

35. Baran, D. et al. Reducing the efficiency-stability-cost gap of organic photovoltaics with highly efficient and stable small molecule acceptor ternary solar cells. Nat. Mater. 16, 363-369 (2017).

36. Zhou, J. Y. et al. Solution-processed and high-performance organic solar cells using small molecules with a benzodithiophene unit. J. Am. Chem. Soc. 135, 8484-8487 (2013).

37. Zhou, J. Y. et al. Small molecules based on benzo[1,2-b:4,5-b ']dithiophene unit for high-performance solution-processed organic solar cells. J. Am. Chem. Soc. 134, 16345-16351 (2012).

38. Tomasic, T. \& Masic, L. P. Rhodanine as a scaffold in drug discovery: a critical review of its biological activities and mechanisms of target modulation. Expert Opin. Drug Dis. 7, 549-560 (2012).

39. Bulic, B. et al. Rhodanine-based tau aggregation inhibitors in cell models of tauopathy. Angew. Chem. Int. Ed. 46, 9215-9219 (2007).

40. Plamont, M. A. et al. Small fluorescence-activating and absorption-shifting tag for tunable protein imaging in vivo. Proc. Natl Acad. Sci. USA 113, 497-502 (2016).

41. Bera, K., Das, A. K., Nag, M. \& Basak, S. Development of a rhodaminerhodanine-based fluorescent mercury sensor and its use to monitor real-time uptake and distribution of inorganic mercury in live zebrafish larvae. Anal. Chem. 86, 2740-2746 (2014).

42. Lee, C. H., Chiang, C. L. \& Liu, S. J. Electrospun nanofibrous rhodanine/ polymethylmethacrylate membranes for the removal of heavy metal ions. Sep. Purif. Technol. 118, 737-743 (2013).

43. Cope, A. C. Condensation reactions. I. The condensation of ketones with cyanoacetic esters and the mechanism of the Knoevenagel reaction. J. Am. Chem. Soc. 59, 2327-2330 (1937).

44. Zhang, Z. et al. Synthesis of polymers with on-demand sequence structures via dually switchable and interconvertible polymerizations. Nat. Commun. 9, 2577 (2018).

45. Zhang, Z. et al. Hybrid copolymerization via mechanism interconversion between radical vinyl-addition and anion ring-opening polymerization. Polym. Chem. 10, 2117-2125 (2019).

46. Kameyama, A., Murakami, Y. \& Nishikubo, T. Novel sequence-ordered polymers by transformation of polymer backbone: Quantitative and regioselective insertion of thiiranes into poly(S-aryl thioester). Macromolecules 32, 1407-1412 (1999).

47. Kudo, H., Makino, S., Kameyama, A. \& Nishikubo, T. Synthesis of cyclic polymers: ring-expansion reaction of cyclic S-dithioester with thiiranes. Macromolecules 38, 5964-5969 (2005).

48. Nagai, A., Koike, N., Kudo, H. \& Nishikubo, T. Controlled thioacyl group transfer (TAGT) polymerization of cyclic sulfide: Novel approach to AB diblock copolymers by the combination of RAFT and TAGT polymerizations. Macromolecules 40, 8129-8131 (2007).

49. Kudo, H., Sato, M., Wakai, R., Iwamoto, T. \& Nishikubo, T. A novel approach to cyclic polysulfides via the controlled ring-expansion polymerization of cyclic thiourethane with thirianes. Macromolecules 41, 521-523 (2008).

50. Kudo, H., Sato, K. \& Nishikubo, T. Controlled insertion reaction of thiirane into carbamothioate: novel synthesis of well-defined polysulfide. Macromolecules 43, 9655-9659 (2010).

51. Xia, Y., Boydston, A. J. \& Grubbs, R. H. Synthesis and direct imaging of ultrahigh molecular weight cyclic brush polymers. Angew. Chem. Int. Ed. 50, 5882-5885 (2011).

52. Bae, I., Han, H. \& Chang, S. Highly efficient one-pot synthesis of Nsulfonylamidines by $\mathrm{Cu}$-catalyzed three-component coupling of sulfonyl azide, alkyne, and amine. J. Am. Chem. Soc. 127, 2038-2039 (2005).

53. Lee, I. H., Kim, H. \& Choi, T. L. Cu-catalyzed multicomponent polymerization to synthesize a library of poly(N-sulfonylamidines). J. Am. Chem. Soc. 135, 3760-3763 (2013).

54. Zhang, Z., You, Y. Z., Wu, D. C. \& Hong, C. Y. Syntheses of sequencecontrolled polymers via consecutive multicomponent reactions. Macromolecules 48, 3414-3421 (2015).

55. Vekariya, R. H. \& Patel, H. D. Recent advances in the synthesis of coumarin derivatives via Knoevenagel condensation: a review. Synth. Commun. 44, 2756-2788 (2014).
56. Bian, Y. J., Qin, Y., Xiao, L. W. \& Li, J. T. New advances of Knoevenagel condensation reactors. Chin. J. Org. Chem. 26, 1165-1172 (2006).

57. Clemens, J. J. et al. Diastereoselective one-pot Knoevenagel condensation/ Corey-Chaykovsky cyclopropanation. J. Org. Chem. 78, 780-785 (2013).

58. Crotti, S. et al. Direct access to alkylideneoxindoles via axially enantioselective Knoevenagel condensation. Org. Lett. 21, 3013-3017 (2019).

59. Kolb, H. C., Finn, M. G. \& Sharpless, K. B. Click chemistry: diverse chemica function from a few good reactions. Angew. Chem. Int. Ed. 40, 2004-2021 (2001).

60. Barner-Kowollik, C. et al. "Clicking” polymers or just efficient linking: what is the difference? Angew. Chem. Int. Ed. 50, 60-62 (2011).

61. Li, M., Liu, C., Hong, C. Y. \& Pan, C. Y. Synthesis of graft copolymer with pendant macrocycles via combination of ATRP and click chemistry. Polymer 71, 23-30 (2015).

62. Wang, D. et al. Highly efficient photosensitizers with far-red/near-infrared aggregation-induced emission for in vitro and in vivo cancer theranostics. Adv. Mater. 30, 1802105 (2018).

63. Wang, D. et al. Rational design of a water-soluble NIR AIEgen, and its application in ultrafast wash-free cellular imaging and photodynamic cancer cell ablation. Chem. Sci. 9, 3685-3693 (2018).

64. Yao, L. et al. Highly efficient near-infrared organic light-emitting diode based on a butterfly-shaped donor-acceptor chromophore with strong solid-state fluorescence and a large proportion of radiative excitons. Angew. Chem. Int. Ed. 53, 2119-2123 (2014)

65. Hu, R., Leung, N. L. C. \& Tang, B. Z. AIE macromolecules: syntheses, structures and functionalities. Chem. Soc. Rev. 43, 4494-4562 (2014).

\section{Acknowledgements}

We acknowledge funding support from the National Key R\&D Program of China (2017YFA0205601), and the National Natural Science Foundation of China (Grant Numbers 21704095, 51625305, 21774113, 21801234, and 21525420).

\section{Author contributions}

Y.Z.Y. and C.Y.H. supervised the study. Z.Z. carried out the experimental measurements, and L.X., X.N., F.W. Z.Y.H., W.J.Z., G.C., W.Q.H., and L.H.W. performed the data analysis. Z.Z., C.Y.H., F.W., Z.Y.H, L.H.W., and Y.Z.Y. wrote the manuscript. All the authors discussed the results and contributed to the manuscript.

\section{Competing interests}

The authors declare no competing interests.

\section{Additional information}

Supplementary information is available for this paper at https://doi.org/10.1038/s41467 020-17474-0.

Correspondence and requests for materials should be addressed to Z.-Y.H., C.-Y.H., L.-H.W. or Y.-Z.Y.

Peer review information Nature Communications thanks Wenxin Wang, Meiran Xie and the other, anonymous, reviewer(s) for their contribution to the peer review of this work. Peer reviewer reports are available.

Reprints and permission information is available at http://www.nature.com/reprints

Publisher's note Springer Nature remains neutral with regard to jurisdictional claims in published maps and institutional affiliations.

Open Access This article is licensed under a Creative Commons Attribution 4.0 International License, which permits use, sharing, adaptation, distribution and reproduction in any medium or format, as long as you give appropriate credit to the original author(s) and the source, provide a link to the Creative Commons license, and indicate if changes were made. The images or other third party material in this article are included in the article's Creative Commons license, unless indicated otherwise in a credit line to the material. If material is not included in the article's Creative Commons license and your intended use is not permitted by statutory regulation or exceeds the permitted use, you will need to obtain permission directly from the copyright holder. To view a copy of this license, visit http://creativecommons.org/ licenses/by/4.0/

(C) The Author(s) 2020 